\title{
ERROR ESTIMATES FOR SOME VARIATIONAL METHODS APPLICABLE TO SCATTERING AND RADIATION PROBLEMS*
}

\author{
BY \\ M. SZAEEK \\ Institute for Fundamental Problems of Technology, Polish Academy of Sciences, Warsaw, Poland
}

\begin{abstract}
We consider variational expressions helpful in calculating the approximate value of a scalar product, in Hilbert space, of an arbitrary vector $g$ with a solution $u^{0}$ of an arbitrary inhomogeneous linear equation. Error bounds for this approximate value are given. For the case where an approximate solution of an inhomogeneous equation is sought in an arbitrary subspace of a space containing $u^{0}$, conditions are specified for a best estimate of the error by the use of two trial vectors. A method is presented for an additional improvement of the error estimate by using four trial vectors.

1. Introduction. In the problems of scattering or radiation we are usually interested in finding out the values of only a few functionals, depending on the solutions of the equations pertinent to the problem. For example, in the problem of scattering on a waveguide junction the quantities of interest are, in most cases, only the amplitudes of the propagating modes, but not the amplitudes of the evanescent modes. When calculating the approximate values of such functionals it may be useful to apply variational methods.
\end{abstract}

To state the problem, consider an equation

$$
k=D u^{0},
$$

where $u^{0} \in \mathrm{H}_{1}, k \in \mathrm{H}_{2}, D \in \mathrm{M}, \mathrm{H}_{1}$ and $\mathrm{H}_{2}$ are Hilbert spaces, and $M$ is the set of linear operators $\left(\mathrm{H}_{1} \rightarrow \mathrm{H}_{2}\right)$. For $g \in \mathrm{H}_{1}$, we want to find the value of the scalar product

$$
\left\langle u^{0}, g\right\rangle \text {. }
$$

That product is assumed to satisfy the conditions for a scalar product in $\mathrm{H}$ spaces.

An approximate value of (2) can be found by using some functionals, such that their stationary values are equal to $\left\langle u^{0}, g\right\rangle$. Let $D^{+}$denote the adjoint of $D$, so that for any $w \in \mathrm{H}_{2}, u \in \mathrm{H}_{1}$ the equality $\langle D u, w\rangle=\left\langle u, D^{+} w\right\rangle$ holds. In general, when $D \neq D^{+}$, as happens for a waveguide junction in the presence of anisotropic media, or where $k \neq$ const $\cdot g$, as is the case if the waveguides at the junction are of different cross-section, the above functionals depend on two trial vectors, $u$ and $w$. We shall consider two simplest functionals of that kind:

$$
\begin{aligned}
& \mathrm{R}_{1}[u, w]=\langle u, g\rangle+\langle k, w\rangle-\left\langle u, D^{+} w\right\rangle, \\
& \mathrm{R}_{1}^{\prime}[u, w]=\frac{\langle u, g\rangle\langle k, w\rangle}{\left\langle u, D^{+} w\right\rangle} .
\end{aligned}
$$

Functional (3) appears in [1], [2], [3] and [4]. Functional (4) was used in [5] and is also equivalent to an expression discussed in [6] in connection with eigenvalue problems. For

* Received January 18, 1967; revised version received January 21, 1969. 
$D=D^{+}$and $g=k$ it reduces to the homogeneous Schwinger's expression

$$
\frac{\langle u, k\rangle\langle k, u\rangle}{\langle u, D u\rangle},
$$

introduced, e.g., in [7] and [8].

In this paper the error bounds for the expressions (3) and (4) are determined (Theorems 2.1 and 2.2). The error estimate for (3) turns out to be better than for (4). These estimates are meaningless when the parameter $m_{0}$, as defined in (6), equals zero, and they may be of little advantage if $m_{0}$ differs only slightly from zero. It is shown in Sec. 4, however, that in such cases one can often obtain a sufficiently large $\mathrm{m}_{0}$ by transforming Eq. (1).

For the case where an approximate solution is sought in an arbitrary subspace $\mathrm{H}_{3} \subset \mathrm{H}_{1}$, conditions are specified to guarantee a best error estimate (Theorem 2.3). Theorem 3.1 allows for an additional improvement by introducing four trial vectors.

2. Error bounds. For any $g, u \in \mathrm{H}_{1}, l, w \in l_{2}$, let

$$
\begin{aligned}
|u| & =[\langle u, u\rangle]^{1 / 2}, \quad|w|=[\langle w, w\rangle]^{1 / 2}, \\
\mathrm{R}_{2}[u] & =\langle k-D u, k-D u\rangle=|k-D u|^{2}, \\
\mathrm{R}_{3}[w] & =\left\langle g-D^{+} w, g-D^{+} w\right\rangle=\left|g-D^{+} w\right|^{2}, \\
\mathrm{~m}_{1} & =\inf _{|u|=1}|D u|, \quad \mathrm{m}_{2}=\inf _{|w|=1}\left|D^{+} w\right|, \quad \mathrm{m}_{0}=\max \left(\mathrm{m}_{1}, \mathrm{~m}_{2}\right),
\end{aligned}
$$

where $\langle z, x\rangle$, for $z, x \in \mathrm{H}_{1}$ or $z, x \in \mathrm{H}_{2}$, is the scalar product in $\mathrm{H}_{1}$ or $\mathrm{H}_{2}$ respectively. We notice that if for every $z \in \mathrm{H}_{2}$ there exists one and only one $x \in \mathrm{H}_{1}$ such that $z=D x$, then $\mathrm{m}_{1}=\mathrm{m}_{2}$, because for $\mathrm{m}_{1}>0$ or $\mathrm{m}_{2}>0$ they are the inverses of the norms of the adjoint operators $D^{-1}$ and $\left[D^{-1}\right]^{+}=\left[D^{+}\right]^{-1}$.

The following theorems can be stated:

Theorem 2.1. Let $D \in \mathrm{M}, g, u \in \mathrm{H}_{1}, k, w \in \mathrm{H}_{2}, 0<\mathrm{m} \leq \mathrm{m}_{0}$. If there exists $u^{0} \in \mathrm{H}_{1}$ satisfying $E q$. (1), and $w^{0} \in \mathrm{H}_{2}$ satisfying the equation

$$
g=D^{+} w^{0}
$$

then

$$
\left|\mathrm{R}_{1}[u, w]-\left\langle u^{0}, g\right\rangle\right| \leq \mathrm{m}^{-1}\left|\mathrm{R}_{2}[u] \mathrm{R}_{3}[w]\right|^{1 / 2}=\mathrm{A}[u, w]
$$

where $\mathrm{A}[u, w]=\mathrm{B}\left|u-u^{0}\right|\left|w-w^{0}\right|$, and $\mathrm{B} \geq 0$ depends only on the directions of the vectors $u-u^{0}$ and $w-w^{0}$.

Proof. Substituting $u=u^{0}+x, w=w^{0}+z$ into $\mathrm{R}_{1}, \mathrm{R}_{2}, \mathrm{R}_{3}$ and taking into account (1) and (7), we obtain

$$
\begin{aligned}
\mathrm{R}_{1}[u, w] & =\left\langle u^{0}, g\right\rangle-\left\langle x, D^{+} z\right\rangle, \\
\mathrm{R}_{2}[u] & =|D x|^{2}, \\
\mathrm{R}_{3}[w] & =\left|D^{+} z\right|^{2} .
\end{aligned}
$$

From (6)

$$
|x| \leq m_{1}^{-1}|D x|, \quad|z| \leq m_{2}^{-1}\left|D^{+} z\right| .
$$


Consequently, by Schwarz' inequality, and from Eqs. (10) and (11), we have

$$
\left|\left\langle x, D^{+} z\right\rangle\right|=|\langle D x, z\rangle| \leq m^{-1}|D x|\left|D^{+} z\right|=m^{-1}\left\{\mathrm{R}_{2}[u] \mathrm{R}_{3}[w]\right\}^{1 / 2} .
$$

This relation, along with (9), proves (8).

Remark. Since we know $D x=D u-k$ and $D^{+} z=D^{+} w-g$, we can sometimes improve the estimate of $|x|$ or $|z|$ as compared to that obtained in the proof of Theorem 2.1. We can replace there $m_{1}$ and $m_{2}$ by $m_{1}^{\prime}$ and $m_{2}^{\prime}$, respectively, where $m_{1}^{\prime}>m_{1}$ or $\mathrm{m}_{2}^{\prime}>\mathrm{m}_{2}$. It can then easily be seen that $\mathrm{m}$ in (8) can be replaced by $\mathrm{m}_{0}^{\prime}=\max \left(\mathrm{m}_{1}\right.$, $\left.\mathrm{m}_{1}^{\prime}, \mathrm{m}_{2}, \mathrm{~m}_{2}^{\prime}\right)$, which may improve the estimate.

In connection with Theorem 2.1 it might be pointed out for comparison that a typical value of a linear estimate of the error in the scalar product (2), as obtained by estimating the error in the approximate solution of the linear equation (1), is $\mathrm{m}^{-1}\left\{\mathrm{R}_{2}[u]\right\}^{1 / 2}|g|$. One can readily see the clear advantage of estimate (8), which is of second order with respect to the errors in $u$ and $w$.

Theorem 2.2. If $\mathrm{m}_{1} \mathrm{~m}_{2}>0,\left\langle u, D^{+} w\right\rangle \neq 0$ then Theorem 2.1 remains true when inequality (8) is replaced by

$$
\left|\mathrm{R}_{1}^{\prime}[u, w]-\left\langle u^{0}, g\right\rangle\right| \leq\left|\left\langle u, D^{+} w\right\rangle\right|^{-1}\left[\left(\mathrm{~m}_{1} \mathrm{~m}_{2}\right)^{-1}|k||g|+\mathrm{m}^{-1}\left|\left\langle u^{0}, g\right\rangle\right|\right]\left|\mathrm{R}_{2}[u] \mathrm{R}_{3}[w]\right|^{1 / 2},
$$

where $\mathrm{R}_{1}^{\prime}[u, w]$ is given by (4).

This can be proved in analogy to Theorem 2.1, taking into account that

$$
\frac{\langle u, g\rangle\langle k, w\rangle}{\left\langle u, D^{+} w\right\rangle}=\left\langle u^{0}, g\right\rangle+\frac{\langle k, z\rangle\langle x, g\rangle-\left\langle u^{0}, g\right\rangle\left\langle x, D^{+} z\right\rangle}{\left\langle u, D^{+} w\right\rangle} .
$$

Using the relation $\langle k, z\rangle\langle x, g\rangle=\left\langle u^{0}, D^{+} z\right\rangle\left\langle D x, w^{0}\right\rangle$, we can also write

$$
\left|\mathrm{R}_{1}^{\prime}[u, w]-\left\langle u^{0}, g\right\rangle\right| \leq\left|\left\langle u, D^{+} w\right\rangle\right|^{-1}\left[\left|u^{0}\right|\left|w^{0}\right|+\mathrm{m}^{-1}\left|\left\langle u^{0}, g\right\rangle\right|\right]\left|\mathrm{R}_{2}[u] \mathrm{R}_{3}[w]\right|^{1 / 2} .
$$

Having in mind that $\left\langle u^{0}, g\right\rangle \cong\left\langle u, D^{+} w\right\rangle$ for $u, w$ approximating closely enough $u^{0}$ and $w^{0}$, we see that estimates (12) and (13) are worse than (8).

Consider now an operator $P_{1}$ projecting every $u \in \mathrm{H}_{1}$ onto a subspace $\mathrm{H}_{3} \subset \mathrm{H}_{1}$ : $P_{1} u \in \mathrm{H}_{3} ; P_{1} P_{1}=P_{1} ; P_{1}^{+}=P_{1}$. In the case where $D$ is a $\mathrm{N} \times \mathrm{N}$ matrix, $P_{1}$ can be defined, e.g., as

$$
\begin{array}{rlrl}
P=\left(P_{\mathrm{ir}}\right) & =\left(\delta_{\mathrm{ir}}\right) & \mathrm{r} & \leq \mathrm{n}_{0} \\
& =(0) & \mathrm{n}_{0}<\mathrm{r} \leq \mathrm{N},
\end{array}
$$

where $\delta_{\mathrm{ir}}$ is the Kronecker delta. Hence an equation $P_{1} k=P_{1} D P_{1} u$, for example, is an approximating set of $\mathrm{n}_{0}$ algebraic equations obtained from the set of $\mathrm{N}$ equations by rejecting $\mathrm{N}-\mathrm{n}_{0}$ equations and setting $\mathrm{N}-\mathrm{n}_{0}$ unknowns equal to zero.

Similarly, let $P_{2}$ be such that for $w \in \mathrm{H}_{2}, \mathrm{H}_{4} \subset \mathrm{H}_{2}$ the relations $P_{2} w \in \mathrm{H}_{4} ; P_{2} P_{2}=$ $P_{2} ; P_{2}^{+}=P_{2}$ hold. We can then formulate

Theorem 2.3. Let $D \in \mathrm{M}, g \in \mathrm{H}_{1}, k \in \mathrm{H}_{2}, u^{\prime} \in \mathrm{H}_{3}, w^{\prime} \in \mathrm{H}_{4}$. If for a certain $u^{\prime 0} \in \mathrm{H}_{3}$

$$
P_{1} D^{+} k=P_{1} D^{+} D P_{1} u^{\prime 0},
$$

then $\mathrm{R}_{2}\left[u^{\prime}\right]$ reaches its least value for $u^{\prime}=u^{\prime 0}$. 
Similarly, if for a certain $w^{\prime 0} \in \mathrm{H}_{4}$

$$
P_{2} D g=P_{2} D D^{+} P_{2} w^{\prime 0},
$$

then $R_{3}\left[w^{\prime}\right]$ reaches its least value for $w^{\prime}=w^{\prime 0}$.

Proof. Substituting $u^{\prime}=u^{\prime 0}+x^{\prime}\left(x^{\prime} \in \mathrm{H}_{3}\right)$ into $\mathrm{R}_{2}\left[u^{\prime}\right]$ we get $\mathrm{R}_{2}\left[u^{\prime}\right]=\left|k-D u^{\prime 0}\right|^{2}+$ $\left|D x^{\prime}\right|^{2}$, which proves the first part of Theorem 2.3. The second part can be proved by substituting $D^{+}, g, w, P_{2}, z$ for $D, k, u, P_{1}, x$, respectively.

Eqs. (14) and (15) are, of course, identical with the equations of the method of least squares, as applied to Eqs. (1) and (7), (see e.g. [9], [10]).

From Theorem 2.3 it follows that estimate (8) is best for $u \in \mathrm{H}_{3}, w \in \mathrm{H}_{4}$ if $u=u^{\prime 0}$, $w=w^{\prime 0}$.

3. Additional estimates. There exists a way of improving estimate (8) if in place of the two trial vectors $u$ and $w$ we consider four vectors. This results from the following theorem in which the spaces $\mathrm{H}_{1}$ and $\mathrm{H}_{2}$ are for simplicity assumed real; this is not an essential restriction since with complex $\mathrm{H}_{1}$ and $\mathrm{H}_{2}$ the problem can always be reduced to that with real $\mathrm{H}_{1}$ and $\mathrm{H}_{2}$.

Theorem 3.1. Let $\mathrm{H}_{1}, \mathrm{H}_{2}$ be real, $D \in \mathrm{M} ; g, u^{0} \in \mathrm{H}_{1} ; k, w^{0} \in \mathrm{H}_{2} ; u^{\prime 0}, x^{\prime} \in \mathrm{H}_{3}$; $z^{\prime}, w^{\prime 0} \in \mathrm{H}_{4}$. If ${u^{\prime \prime}}^{\prime 0}$ and $w^{\prime 0}$ satisfy (14) and (15), respectively, then

$$
\max _{x^{\prime}, z^{\prime}}\left(\mathrm{a}_{1}-\mathrm{a}_{2}\right) \leq\left\langle u^{0}, g\right\rangle \leq \min _{x^{\prime}, z^{\prime}}\left(\mathrm{a}_{1}+\mathrm{a}_{2}\right)
$$

where

$$
\begin{aligned}
& \mathrm{a}_{1}=\mathrm{R}_{1}\left[{u^{\prime 0}}^{\prime}, w^{\prime 0}\right]+\left\langle x^{\prime}, g-D^{+} w^{\prime 0}\right\rangle+\left\langle k-D u^{\prime 0}, z^{\prime}\right\rangle-\left\langle x^{\prime}, D^{+} z^{\prime}\right\rangle, \\
& \mathrm{a}_{2}=\mathrm{m}^{-1}\left\{\mathrm{R}_{2}\left[{u^{\prime \prime}}^{\prime}\right]+\left\langle x^{\prime}, D^{+} D x^{\prime}\right\rangle\right\}^{1 / 2}\left\{\mathrm{R}_{3}\left[w^{\prime 0}\right]+\left\langle z^{\prime}, D D^{+} z^{\prime}\right\rangle\right\}^{1 / 2} .
\end{aligned}
$$

Proof. Let $u=u^{\prime 0}+x^{\prime}, w=w^{\prime 0}+z^{\prime}$. Taking into account relations (14) and (15) we get $\mathrm{a}_{1}=\mathrm{R}_{1}[u, w], \mathrm{a}_{2}=\mathrm{m}^{-1}\left|\mathrm{R}_{2}[u] \mathrm{R}_{3}[w]\right|^{1 / 2}$. Hence, inequality (8) can be written in the form

$$
\mathrm{a}_{1}-\mathrm{a}_{2} \leq\left\langle u^{0}, g\right\rangle \leq \mathrm{a}_{1}+\mathrm{a}_{2} .
$$

This is valid for every $x^{\prime} \in \mathrm{H}_{3}, z^{\prime} \in \mathrm{H}_{4}$, which proves (16).

For $x^{\prime}=z^{\prime}=0$ inequality $\left(16^{\prime}\right)$ goes into (8) with $u={u^{\prime}}^{0}, w=w^{\prime 0}$. Inequality (16) gives, therefore, an estimate not worse than (8) with $u \in \mathrm{H}_{3}, w \in \mathrm{H}_{4}$.

The exact values of $\max \left(a_{1}-a_{2}\right)$ and $\min \left(a_{1}+a_{2}\right)$ apparently are not worth searching for in view of mathematical difficulties. The method of steepest descent can be applied here. This corresponds to setting $z^{\prime}=\mathrm{d}_{1} P_{2} y_{1} ; x^{\prime}=\mathrm{d}_{2} P_{1} y_{2}$, where $\mathrm{d}_{1}, \mathrm{~d}_{2}$ are real numbers, $y_{1}=k-D u^{\prime 0}, y_{2}=g-D^{+} w^{\prime 0}$. One can also put $z^{\prime}=\mathrm{d}_{1}^{\prime} y_{1}^{\prime}, x^{\prime}=\mathrm{d}_{2}^{\prime} y_{2}^{\prime}$, where $y_{1}^{\prime} \in \mathrm{H}_{4}$ and $y_{2}^{\prime} \in \mathrm{H}_{3}$ are the eigenvectors belonging to the lowest eigenvalues of the operators $P_{2} D D^{+} P_{2}$ and $P_{1} D^{+} D P_{1}$, respectively. Such a choice will guarantee the slowest increase of $a_{2}$ with the increase of $\left|d_{1}^{\prime}\right|$ and $\left|d_{2}^{\prime}\right|$. Combining both the substitutions we can put $z^{\prime}=\mathrm{d}_{1} P_{2} y_{1}+\mathrm{d}_{1}^{\prime} P_{2} y_{1}^{\prime} ; x^{\prime}=\mathrm{d}_{2} P_{1} y_{2}+\mathrm{d}_{2}^{\prime} P_{1} y_{2}^{\prime}$.

Applying the method of steepest descent we obtain from (16) the inequality

$$
\max _{d_{1}, d_{\mathbf{d}}}\left(a_{1}-a_{2}\right) \leq\left\langle u^{0}, g\right\rangle \leq \min _{d_{1}, d_{2}}\left(a_{1}+a_{2}\right),
$$

where 


$$
\begin{aligned}
& \mathrm{a}_{1}=\mathrm{R}_{1}\left[u^{\prime 0}, w^{\prime 0}\right]+\mathrm{d}_{1}\left|P_{2} y_{1}\right|^{2}+\mathrm{d}_{2}\left|P_{1} y_{2}\right|^{2}-\mathrm{d}_{1} \mathrm{~d}_{2}\left\langle y_{2}, P_{1} D^{+} P_{2} y_{1}\right\rangle, \\
& \mathrm{a}_{2}=\mathrm{m}^{-1}\left\{\left|y_{1}\right|^{2}+\mathrm{d}_{2}^{2}\left|D P_{1} y_{2}\right|^{2}\right\}^{1 / 2}\left\{\left|y_{2}\right|^{2}+\mathrm{d}_{1}^{2}\left|D^{+} P_{2} y_{1}\right|^{2}\right\}^{1 / 2},
\end{aligned}
$$

because $\mathrm{R}_{2}\left[{u^{\prime}}^{0}\right]=\left|y_{1}\right|^{2}, \mathrm{R}_{3}\left[{w^{\prime}}^{0}\right]=\left|y_{2}\right|^{2}$. Knowing $D_{1}=P_{1} D^{+} D P_{1}$ and $D_{2}=P_{2} D D^{+} P_{2}$, needed in (14) and (15), we can express here $\left|D P_{1} y_{2}\right|^{2}=\left\langle y_{2}, P_{1} D_{1} P_{1} y_{2}\right\rangle ;\left|D^{+} P_{2} y_{1}\right|^{2}=$ $\left\langle y_{1}, P_{2} D_{2} P_{2} y_{1}\right\rangle$.

The functions $a_{1}$ and $a_{2}$ depend on two variables, $d_{1}$ and $d_{2}$. For such functions it is relatively easy to calculate their approximate extreme values. Apart from that, to determine the coefficients defining $a_{1}$ and $a_{2}$ it is sufficient to calculate some products of "small" ( $\mathrm{n}_{0}$-dimensional) vectors with matrices, since all time-consuming calculations have been already performed in connection with Eqs. (14), (15) and (8). It therefore seems reasonable to use estimate (17), even if there is only a relatively small chance that this might essentially improve the error estimate.

So far, this author knows of only one case where estimate (17) has been applied; this was in numerical calculations concerning scattering on a waveguide junction in the presence of an anisotropic medium. In that case no noticeable improvement in the error estimate was obtained despite the fact that the necessary conditions, as given below, for estimate (17) to be useful were satisfied.

In order to find the above-mentioned conditions consider a quantity $a_{3}$ defined as the ratio of the shift in the upper bound of the error to the distance between the bounds

$$
\begin{aligned}
\mathrm{a}_{3} & =\left[\left.\left(\mathrm{a}_{1}+\mathrm{a}_{2}\right)\right|_{\mathrm{d}_{1}=\mathrm{d}_{3}=0}-\min _{\mathrm{d}_{1}, \mathrm{~d}_{2}}\left(\mathrm{a}_{1}+\mathrm{a}_{2}\right)\right]\left[2 \mathrm{a}_{2}||_{\mathrm{d}_{1}=\mathrm{d}_{2}=0}\right]^{-1} \\
& =\frac{1}{2} \max _{\mathrm{d}_{1}, \mathrm{~d}_{2}}\left[-\mathrm{d}_{1}\left|P_{2} y_{1}\right|^{2}-\mathrm{d}_{2}\left|P_{1} y_{2}\right|^{2}+\mathrm{d}_{1} \mathrm{~d}_{2}\left\langle y_{2}, P_{1} D^{+} P_{2} y_{1}\right\rangle+\mathrm{m}^{-1}\left|y_{1}\right|\left|y_{2}\right|-\mathrm{a}_{2}\right] \frac{\mathrm{m}}{\left|y_{1}\right|\left|y_{2}\right|} .
\end{aligned}
$$

This quantity can serve as a measure of the achieved improvement in the error estimate. The inequality $0 \leq a_{3} \leq 1$ must be satisfied. The larger $a_{3}$, the greater the improvement in the estimate. We always have $\left|y_{1}\right|^{2} \geq\left|P_{2} y_{1}\right|^{2},\left|y_{2}\right|^{2} \geq\left|P_{1} y_{2}\right|^{2},\left|D P_{1} y_{2}\right| \geq \mathrm{m}_{1}\left|P_{1} y_{2}\right|$, $\left|D^{+} P_{2} y_{1}\right| \geq \mathrm{m}_{2}\left|P_{2} y_{1}\right|$.

Taking for example $\left|y_{1}\right|=\left|y_{2}\right|, \mathrm{b}\left|y_{1}\right|=\left|P_{2} y_{1}\right|=\left|P_{1} y_{2}\right|, \mathrm{m}_{0}=\mathrm{m},\left|D P_{1} y_{2}\right|=\left|D^{+} P_{2} y_{1}\right|$ $=\mathrm{nm}\left|y_{1}\right|,\left\langle y_{2}, P_{1} D^{+} P_{2} y_{1}\right\rangle=0, \mathrm{~d}_{1}=\mathrm{d}_{2}, \mathrm{n} \geq \mathrm{b}, 0 \leq \mathrm{b} \leq 1$, we obtain

$$
a_{3}=\frac{m}{2} \max _{d_{1}}\left[-2 d_{1} b^{2}-d_{1}^{2} n^{2} m\right] \text {. }
$$

The maximum is reached for $d_{1}=-b^{2} / n^{2} m$; then $a_{3}=b^{4} / 2 n^{2}$. Hence the outlined method based on Theorem 3.1 can be sensibly applied only when $\mathrm{n}$ can be small, e.g. $\mathrm{b} \leq \mathrm{n} \leq 2$, and, simultaneously, $b \cong 1$. This can be the case only when $\mathrm{m}_{1}^{\prime \prime} / \mathrm{m}_{0}<2,5$ and $\mathrm{m}_{2}^{\prime \prime} / \mathrm{m}_{0}<2,5$, where

$$
\mathrm{m}_{1}^{\prime \prime}=\inf _{\left|P_{1} u\right|=1}\left|D P_{1} u\right| \quad \mathrm{m}_{2}^{\prime \prime}=\inf _{\left|P_{2} w\right|-1}\left|D^{+} P_{2} w\right|
$$

with $u \in \mathrm{H}_{1}, w \in \mathrm{H}_{2}$. These conditions remain also valid in a more general case, in particular, in every case where $\left\langle y_{2}, P_{1} D^{+} P_{2} y_{1}\right\rangle=0$.

4. Remarks concerning $m_{0}$ and $A[u, w]$. In connection with the estimates given it is useful to examine how transformations of Eq. (1), with $\mathrm{m}_{0}=0$ or $\mathrm{m}_{0}$ approaching zero, may influence the values of $\mathrm{m}_{0}$ and $\mathrm{A}[u, w]$ in (8).

Suppose that Eq. (1) is equivalent to an infinite set of algebraic equations. Usually 
this set can be approximated, with an accuracy sufficient for the physical problem under consideration, by a finite set of $\mathrm{N}$ algebraic equations with $\mathrm{m}_{0} \neq 0$. This procedure is analogous to that where Eq. (1) is transformed by multiplying it by such a linear operator $L$ that for the operator $L D$ the value of $\mathrm{m}_{0}$ is positive. To obtain a suitably large $\mathrm{m}_{0}$ we can also apply these two procedures successively. Let $\mathrm{A}_{1}[u]=\mathrm{m}_{0}^{-1}\left\{\mathrm{R}_{2}[u]\right\}^{1 / 2}$. Putting for simplicity $\mathrm{m}=\mathrm{m}_{0}$, we have $\mathrm{A}[u, w]=\mathrm{A}_{1}[u]\left\{\mathrm{R}_{3}[w]\right\}^{1 / 2}$. In Theorem 4.1 we shall specify the operators $L$ for which $A_{1}[u]$ reaches a minimum.

Suppose that $D$ can be written in a matrix representation as a finite $\mathrm{N} \times \mathrm{N}$ matrix $\left(D_{\mathrm{ir}}\right)$, with $\mathrm{m}_{0} \neq 0$. Let $\mathrm{H}_{1}=\mathrm{H}_{2}$, det $(D) \neq 0$, det $(L) \neq 0$. We have in place of Eq. (1) an equation $L k=L D u^{0}$. Thus $\mathrm{R}_{2}[L, u]=|L(k-D u)|^{2}, \mathrm{~m}_{0}(L)=\min _{|u|=1}|L D u|$, $\mathrm{A}_{1}[L, u]=\left\{\mathrm{m}_{0}(L)\right\}^{-1}\left\{\mathrm{R}_{2}[L, u]\right\}^{1 / 2}$. Let the components of an arbitrary vector $x \in \mathrm{H}_{1}$ be $\mathrm{x}_{\mathrm{i}}(\mathrm{i}=1,2, \cdots, \mathrm{N})$, and $\langle x, t\rangle=\sum_{\mathrm{i}=1}^{\mathrm{N}} \mathrm{x}_{\mathrm{i}}^{*} \mathrm{t}_{\mathrm{i}}$ for any $x, t \in \mathrm{H}_{1}$ where $\mathrm{x}_{i}^{*}$ denotes the complex conjugate of $x_{i}$. We can always adopt such a definition of a scalar product, defining accordingly the vector $g$. Let $u^{0}, u, x \in \mathrm{H}_{1} ; u=u^{0}+x$.

Theorem 4.1. If $\operatorname{det}(D) \neq 0$, $\operatorname{det}(L) \neq 0, \mathrm{H}_{1}=\mathrm{H}_{2}$, then for every $u \in \mathrm{H}_{1}$ the quantity $\mathrm{A}_{1}[L, u]$ assumes a smallest value for $L D=\mathrm{c} S$ where $\mathrm{c}$ is any number different from zero and $S$ is any unitary matrix.

Proof.

$$
\mathrm{A}_{1}[L, u]=\left[\mathrm{m}_{0}(L)\right]^{-1}\left[\left\langle x,[L D]^{+} L D x\right\rangle\right]^{1 / 2} .
$$

Since $L_{1}=[L D]^{+} L D$ is a Hermitian positive definite matrix we can pass by means of a unitary transformation $S_{1}$ to a coordinate system where $L_{1}$ is diagonal and can be written $L_{1}=\left(\mathrm{a}_{\mathrm{i}}^{2} \delta_{\mathrm{ir}}\right)$, where $\mathrm{a}_{\mathrm{i}}>0$. In this new coordinate system the definition of the scalar product remains unchanged and $\mathrm{m}_{0}(L)=\min _{\mathrm{i}} \mathrm{a}_{\mathrm{i}}$. Hence

$$
\mathrm{A}_{1}[L, u]=\left\{\sum_{\mathrm{i}=1}^{\mathrm{N}}\left|\mathrm{x}_{\mathrm{i}}\right|^{2} \mathrm{a}_{\mathrm{i}}^{2}\right\}^{1 / 2} \frac{1}{\min _{\mathrm{i}} \mathrm{a}_{\mathrm{i}}} .
$$

Thus $\mathrm{A}_{1}[L, u]$ assumes a smallest value for $\mathrm{a}_{\mathrm{i}}=$ const $(\mathrm{i}=1,2, \cdots, \mathrm{N})$. This is so if $L D=$ c $S$, e.g. if $L=D^{-1}$.

By making $\mathrm{N}$ tend to infinity, Theorem 4.1 can be generalized to the case of infinite matrices, even with $\operatorname{det}(D)=0$, provided that to every $z \in \mathrm{H}_{2}$ there corresponds only one $x \in \mathrm{H}_{1}$ such that $z=D x$.

Regarding $\mathrm{R}_{3}[w]$ the following facts can be noted. In place of Eq. (7) we have $g=$ $D^{+} L^{+} w^{0}(L)$. Hence $\mathrm{R}_{3}[L, w(L)]=\left|g-D^{+} L^{+} w(L)\right|^{2}$. We can expect that the closer $L D$ approaches c $S$, the smaller $\mathrm{R}_{3}[L, w(L)]$, because it is then easier to find a good approximation to the solution of the equation $L D g=L D[L D]^{+} w^{0} \cong|\mathrm{c}|^{2} w^{0}$.

We can, therefore, in general say that the closer $L D$ approaches $\mathrm{c} S$, that is the less the norm of $L D$ differs from $\mathrm{m}_{0}(L)$, the smaller $\mathrm{A}[u, w]$. We can also notice that, in accordance with the inequalities given at the end of Sec. 3, most favorable conditions for applying formulas (16) and (17) exist when $L D \cong \mathrm{c} S$, as in that case the norm of $L D$ differs little from $\mathrm{m}_{0}(L)$.

If $D$ does not differ much from a diagonal matrix we can, in particular, set $L=$ $\left(\mathrm{c}_{\mathrm{i}} \delta_{\mathrm{ir}}\right)$ with suitably chosen numbers $\mathrm{c}_{\mathrm{i}}$. Assuming $L$ in that form may also turn out useful in other cases.

In connection with our estimates the question arises of determining the number $\mathbf{m}_{0}$. 
This question will not be discussed here. It may, however, be noticed that even the knowledge of a very rough approximation of $m_{0}$ will be useful. In addition, if we want to find the values of $\mathrm{m}_{0}$ for many operators $D$ or $L D$ not much different one from another, it is sufficient to find them for some of these operators and apply to the rest the estimates connecting the numbers $m_{0}$ of two operators and the norm of their difference (cf. e.g. [11]). If the operator $L D$ is given in a matrix form and that matrix is not much different from diagonal, we can also apply other estimates such as the Hadamard estimate [11].

\section{References}

[1] P. Roussopoulos, Methodes variationnelles en theorie des collisions, C. R. Acad. Sci. Paris 236, 18581860 (1953).

[2] M. Becker, The principles and application of variational methods, Research monograph No. 27, MIT Press, Cambridge, Massachusetts.

[3] G. Pomraning, A variational principle for linear systems, J. Soc. Indust. Appl. Math. 13, 511 (1965).

[4] W. W. Nikol'skij, Variacionnye metody dlja vnutrennych zadač élektrodynamiki (Variational methods for electromagnetic boundary-value problems), "Nauka", Moskva 1967, p. 25.

[5] L. A. Vajnštejn, Volny toka v tonkom cil'indričeskom provodnike, III. Variacionnyj metod (Waves of current in a thin cylindrical conductor, III. Variational method), J. Tech. Phys. 31, 29-44 (1961).

[6] L. Cairo and T. Kahan, Techniques variationnelles en radioélectricité, Dunod, Paris 1962, p. 54.

[7] F. E. Borgnis and C. H. Papas, Randwertprobleme der Mikrowellenphysik, Springer-Verlag, Berlin 1955.

[8] R. E. Collin, Field theory of guided waves, McGraw-Hill, New York, 1960, p. 314-363.

[9] S. G. Michlin, Variacionnye metody $v$ matematičeskoj fizike (Variational methods in mathematical physics), GITTL, Moskva 1957, pp. 410-417.

[10] M. Altman, Metody przybliżone analizy funkcjonalnej (Approximate methods of functional analysis), Zakład Narodowy im. Ossolińskich, Wydawnictwo PAN, Wrocław-Warszawa-Krak6w 1963, pp. 35-38.

[11] E. Bodewig, Matrix calculus, North-Holland, Amsterdam, 1956, pp. 57-69. 